\title{
Marketing RH: La Marque Emoployeur/ un Levier pour se Demarquer
}

\section{Idrissi Khadija, Professeur de L'enseignement Supérieur \\ Hanaty Chorine, Doctorante \\ Senihji Khadija, Doctorante}

Université Hassan, Laboratoire de Recherche en Management et

Développement, Settat, Maroc

Doi:10.19044/esj.2019.v15n22p102 URL:http://dx.doi.org/10.19044/esj.2019.v15n22p102

\section{Résumé}

Aujourd'hui dans un monde dominé par le digital, c'est tout naturel que les entreprises aient intégré des nouveaux modes de travail façonnés par le numeriqué.Cette mutation digitale a modifié le travail des gestionnaires et les a amenés à repenser une méthodologie devenue obsoleté. Et tout état de cause, La fonction RH ne s'échappe pas à cette vague de digitalisation qui a submergé toutes les autres fonctions de l'entreprise y compris le marketing. Emportée par le digital, la fonction RH est passée d'une fonction support à une fonction de partenaire stratégique. Ce passage a marqué l'avènement du «E-marketing RH », un mariage inattendu qui a bouleversé la donne. Dès lors, Le marketing RH est une approche rénovée de la GRH, qui cherche d'une part à améliorer l'attractivité interne de l'entreprise et d'autre part développer son attractivité externe. Nous nous intéressons dans cet article à l'attractivité externe de l'entreprise. En effet pour attirer les candidats l'entreprise est appelée à polir sa marque employeur en véhiculant sa culture. La relation entre la marque employeur et l'e-recrutement sera donc le sujet de notre article. Notre réflexion est construite en quatre étapes : nous verrons en premiers temps la genèse du marketing $\mathrm{RH}$ et son rôle dans le développement de la marque employeur. Secundo nous mettons en surbrillance l'usage du web 2.0 dans la construction de la marque employeur numérique et son impact sur l'erecrutement. In fine, nous présentons quelques stratégies de recrutement digital de certaines Multinationales.

Mots-clés: Marketing RH, E-marque employeur, E-recrutement, Attractivité 


\title{
The HR Marketing: The Employer Brand/ a Lever to Stand up
}

\author{
Idrissi Khadija, Professeur de L'enseignement Supérieur \\ Hanaty Chorine, Doctorante \\ Senihji Khadija, Doctorante
}

Université Hassan, Laboratoire de Recherche en Management et

Développement, Settat, Maroc

\begin{abstract}
Today, in a world dominated by digital technology, it is obvious that companies have to integrate new methods of getting work done. This digitalization has changed the work of managers and have made them realized that their methodology has become obsolete. At any rate, the HR function indeed was impacted by this wave of digitization that has overwhelmed all other functions of the company including marketing. Driven by digital technology, the HR function has moved from a support function to a business partner function. This switch marked the advent of the "E-marketing HR", an unexpected marriage that upset the situation. Since then, HR marketing is a renewed approach of HRM, which seeks, on the one hand, to improve the internal attractiveness of the company. On the other hand, it helps to develop the external attractiveness of the company. Thus, this paper focuses on the external attractiveness of the company. To attract candidates, the company needs to focus on improving their brand by communicating its culture. The relationship between the digital employer brand and e-recruitment will therefore be the subject of our article. Our thinking is built on four stages: First we will consider the genesis of HR marketing and its role in the development of the employer brand. The second part will be devoted to the use of web 2.0 in the construction of a digital employer brand and its impact on e-recruitment. Finally, we will present some digital recruitment strategies of some multinationals.
\end{abstract}

Keywords: HR Marketing, Employer brand, E-recruitment, Attractiveness

\section{Introduction}

Dans un contexte marqué par la raréfaction des compétences et la guerre des talents, les organisations sont invitées à repenser leurs modes de management et leurs façons d'appréhender leurs collaborateurs. Les modes de 
gestion d'hier ne sont plus adaptés pour piloter les organisations d'aujourd'hui et de demain.

Les collaborateurs et/ou candidats potentiels expriment de plus en plus des attentes nouvelles auxquelles il faut savoir répondre. L'impact du digital conduit également à envisager de nouvelles modalités de travail.

Ces mutations conduisent à rapprocher deux concepts éloignés : celui de la GRH et celui du marketing.

En effet, considérées comme deux disciplines distinctes des sciences de gestion, la GRH et le Marketing se trouvent aujourd'hui associés pour mieux appréhender le pilotage des personnes et des organisations (Brillet\&Gavoille, 2017).

Ce mariage n'est pas accepté par tout le monde et ceci explique pourquoi certains le rejettent et d'autres l'associent à de simples opérations de communication et de publicité destinées à améliorer l'image des organisations et leur chiffre d'affaires.

Loin de cette vision, nous considérons que cette approche marketing RH est une réponse possible face aux mutations des marchés, aux transformations des organisations, à l'évolution des attitudes face au travail.

Il est à rappeler que pendant ces deux dernières décennies des profondes mutations sont survenues sur le monde économique et technologique. Parmi la plus remarquables on cite : la digitalisation.

En effet, l'apparition de la digitalisation a coïncidé avec la naissance de la génération «Y $\mathrm{Y}$, une génération familiarisée avec le numérique.

D'ici, on peut dire que la première fonction impactée par la digitalisation est le marketing $\mathrm{RH}$.

Cette dernière a tendance aujourd'hui à digitaliser toutes ses pratiques pour répondre aux attentes de cette génération connectées.

Nous allons montrer à partir de cet article comment le marketing RH développe la stratégie de e-marque employeur et comment celle-ci impacte l'attractivité de l'entreprise en matière de recrutement.

Nous formulons ainsi l'hypothèse selon laquelle la marque employeur peut avoir un impact sur l'attractivité de l'entreprise et le recrutement des talents.

Cette orientation de la réflexion met en valeur le paradigme positiviste puisqu'il s'agit d'appréhender et de comprendre des pratiques originales et réels mises en œuvre dans un contexte peu initié.

Pour ce faire on abordera de prime abord la genèse du marketing RH, ensuite nous allons apporter un éclairage sur la notion du e-marketing $\mathrm{RH}$.

L'article traitera en second lieu la notion de la marque employeur numérique et sa relation avec l'e-recrutement.

La dernière partie sera consacrée à la présentation des stratégies du erecrutement adoptées par quelques entreprises multinationales. 


\section{La Genese Du Marketing RH : Un Mariage Inedit}

a. Le marketing RH

Aujourd'hui, dans un marché qualifié de raréfaction des ressources humaines, Le mariage entre marketing et GRH devient une affaire inévitable. Les entreprises sont amenées non seulement à optimiser leurs ressources humaines, mais également à utiliser des techniques du marketing vers une logique de «cliemployé » considérant ainsi le salarié comme un individu à part entière.

On voit d'emblée que le marketing s'applique dès lors qu'il y a un échange entre deux parties.

Partant de la même logique, la GRH correspond aussi à un échange entre le salarié et l'employeur d'ici, il parait logique de parler du marketing RH.

De par ces constats, on peut esquisser les postulats suivants :

- L'homme n'est plus une charge à minimiser, il est plutôt une variable stratégique qu'il faut fidéliser, motiver et attirer. Il est le premier client de l'entreprise au sens le plus noble pour reprendre l'expression de LIGER.

- Le marketing RH n'est pas cantonné à la vente, il consiste à charmer les candidats, fidéliser les employés et développer la marque employeur.

- On distingue ainsi le marketing RH interne relatif à la fidélisation des salariés et le marketing RH externe relatif au Branding, recrutement.

- La démarche mercatique dispose d'une panoplie d'outils adaptable à la GRH.

Panczuk et Point (2008) insistent sur le fait que le marketing RH ne se limite pas à la publicité ou la vente mais, consiste à mettre en œuvre une stratégie en se basant sur une segmentation du marché à conquérir, un positionnement de la gamme de produits $\mathrm{RH}$, et une transcription d'une véritable démarche marketing mix adaptée au champ des ressources humaines.

De facto il y a plusieurs raisons pour l'adopter une démarche du marketing $\mathrm{RH}$ :

- un taux de turn-over important engendré par l'aspiration à de meilleures conditions de travail ; voire un zapping professionnel de la génération $\mathrm{Y}^{2}$;

- l'évolution de la relation au travail : les salariés deviennent plus volatiles et plus exigeants. Ils ne sont plus uniquement intéressés par une rémunération attrayante, mais aussi par des perspectives de

\footnotetext{
${ }^{2}$ La génération Y désigne une catégorie de personnes occidentales, nées entre 1980 et 2000. C'est une génération liée au Web et aux nouvelles technologies.
} 
développement professionnel et un grand équilibre vie privée/vie professionnelle.

Selon Liger (2008), le marketing RH est « une nouvelle approche de la relation salariélentreprise qui consiste à considérer les collaborateurs comme des clients au sens le plus noble».

Liger (2016) ajoute "les RRH doivent se servir des techniques issues du marketing, pour attirer le futur collaborateur (le séduire), l'intégrer (l'accueillir) et le garder (le fidéliser), à l'instar d'un client d'une marque qui serait dans ce cas celle de l'entreprise employeur ».

On y retrouve bien ici la notion du salarié/client sur lequel on applique les techniques du marketing traditionnel.

\section{b. Le E-marketing RH}

Avec l'essor des nouvelles technologies, le marketing traditionnel s'est étendu au marketing digital, les Ressources Humaines se digitalisent également avec l'évolution des méthodes et canaux de recrutement. On voit donc depuis quelques mois se développer l'E-marketing RH, dont le but est d'améliorer l'attractivité de l'entreprise à travers sa marque employeur. ${ }^{3}$

Aujourd'hui le monde digital a changé la donne. Il a équilibré le rapport entre entreprises et candidats, dans le sens où ces derniers, s'ils sont issus de la génération $\mathrm{Y}$, inversent les rôles et deviennent des candidateurs. Cela veut dire qu'au lieu de se limiter aux messages de recrutement émis par l'entreprise, ces derniers se renseignent sur la réputation de celle-ci et de celle des concurrents pour croiser les données (benchmark), et ce en s'outillant entre autres des réseaux sociaux.

Les données collectées par les candidats, sont issues généralement des clients, collaborateurs, partenaires, fournisseurs, et même des stagiaires.

Ces données vont permettre aux candidats de construire une image sur l'entreprise et sur sa réputation, d'ici on remarque l'importance de la marque employeur et son impact sur l'attractivité et le recrutement des talents.

L'entreprise qui souhaite recruter les talents BOB (best of the best) doit bien façonner son image, communiquer sur ses valeurs, partager l'expérience de ses collaborateurs dans le but de construire une carte d'identité différente et séduisante.

\section{La Marque Employeur : Un Outil D'attractivite De L'entreprise}

La marque employeur a été définie par différents auteurs, elle a été citée pour la première fois par Barrow (1996), qui l'a défini comme

\footnotetext{
${ }^{3}$ La marque employeur est un groupement d'actions marketing activés dans le but de rendre l'entreprise attractive aux yeux des futurs collaborateurs et de fidéliser les salariés actuels.
} 
"l'ensemble des avantages fonctionnels, économiques et psychologiques des emplois que propose une entreprise en tant qu'employeur $\gg$.

Cette définition a été complétée par Panczuk et Point (2008) pour mettre en évidence l'importance du rôle des salariés, mais également celui des autres parties prenantes.

Selon eux, la marque employeur est « un processus par lequel les salariés internalisent l'image de marque désirée et sont motivés pour projeter cette image aux clients et autres parties organisationnelles ».

On retient celle de Liger (2008), pour lui il s'agit, de développer une relation durable et de plus en plus individualisée avec les collaborateurs. Il s'agit également de faire de l'entreprise un employeur attractif et de son nom une véritable marque, associée à de vraies valeurs.

Pour La Pinta (2011) la marque employeur est définie avec ces quatre éléments :

- Ce que votre entreprise est vraiment ;

- Ce que vous dites de votre entreprise ;

- Ce que vos collaborateurs disent de votre entreprise ;

- Ce que le public dit de votre entreprise (réputation employeur).

En grosso modo la marque employeur est un moyen pour se faire connaître comme un employeur de référence, elle permet à l'entreprise de communiquer sur sa culture, ses promesses et ses valeurs.

Autrement dit, il s'agit d'une profonde connaissance de l'entreprise, de son histoire, de sa culture, de ses collaborateurs... «Il s'agit d'identifier ses forces et ses faiblesses pour ensuite pouvoir mettre en avant une identité forte et originale, et non pas faire uniquement la promotion de valeurs évidentes et communes à toutes les organisations comme le respect de l'environnement ou de l'humain » (Fougerat, 2010).

C'est en développant l'image employeur, qu'on augmente les attentes des candidats. Or si cette marque employeur n'est pas en phase avec la réalité, celle-ci peut créer un décalage entre la promesse de l'embauche et ce qui est vécu. Ce qui provoquera l'effet inverse, à savoir entrainer des départs des salariés au lieu de les fidéliser.

Dans ce cas, les promesses véhiculées par l'entreprise dans les annonces de recrutement doivent être en cohérence avec la réalité vécue par les salariés, car la génération « j' ai le droit » est une génération curieuse qui ne va pas tarder à chercher les non-dits par l'entreprise sur les forums, blog ou réseaux sociaux.

\section{a. E-marque employeur}

Le web au départ était un outil passif et « unidirectionnel » avec un internaute qui était seulement un consommateur de contenus. Ce dernier n'avait aucune latitude pour interagir avec l'information qu'il consultait. 
Avec l'arrivée des réseaux sociaux, les entreprises ne sont plus les seules à communiquer sur leurs marques et leurs valeurs, mais aussi les collaborateurs, les clients...Tout le monde peut échanger son point de vue notamment sur Facebook, Twitter, blog, forum de discussion.

Selon Anna (2011), «le Web 2.0 a permis à l'internaute de devenir "consom'acteur" » qui publie partage et communique des informations, ceci a profondément changé la relation du candidat avec l'entreprise, et l'a poussé à soigner son image sur le web.

La marque employeur est un multicanal et utilise différents outils de communication. On retrouve ci-dessous un aperçu de la diversité des outils 2.0 :

- Un site Carrière avec de l'information sur les postes et les profils recherchés, la présentation des valeurs de l'Entreprise et même des conseils pour les Candidats comme 1'Espace Coaching de la Société Générale.

- Un site de questions-réponses sur les métiers de l'Entreprise comme le site «Backstage» que vient de lancer BNPParibas.

- Un blog pour donner une image plus humaine et plus conviviale et aborder des thématiques capitales sur lesquelles la marque est engagée : handicap, égalité hommes- femmes.

- Une page sur Linkedin.

- Une page sur Facebook sur lesquelles on peut trouver un espace de discussion (le mur), de l'information et même des offres d'emploi.

- Un compte Twitter.

- Une Chaîne sur Youtube relais des valeurs et engagements de la marque qui présente les métiers de l'entreprise au travers les collaborateurs qui les exercent.

- Une Web TV avec différentes chaînes thématiques comme celle de la Société Générale (http://careers.societegenerale.tv/) qui présente en images les métiers, les parcours, le groupe, les engagements, en donnant la parole à ses collaborateurs ou à des experts.

- Des représentants RH portant les valeurs de l'Entreprise via leurs profils sur les réseaux sociaux comme Viadeo et Linkedin, et leurs comptes Twitter.

L'ensemble de ces outils nécessitent un pilotage, d'où l'importance du community manager, un nouveau métier RH, c'est un manager RH capable de parler de tous les métiers de l'entreprise.

Son rôle est de maîtriser et piloter la marque en interne comme en externe, il représente et augmente les interactions avec la marque sur le web. 
Par exemple, un community manager va animer les discussions au sein de forums, de blogs,...et également participer aux discussions qui se déroulent sur d'autres sites afin d'y répondre au mieux. C'est lui qui va également poster des commentaires sur les blogs, créer et animer une page Facebook, un groupe sur Viadeo ou Linked In, ou bien encore du contenu sur YouTube ou Slideshare.

Ainsi, pour se faire une idée sur l'e-marque employeur en termes de volume, voici quelques chiffres qui devraient aider à mieux appréhender l'ampleur de ce phénomène :

Selon une étude réalisée en 2011 par le cabinet de conseil en marketing $\mathrm{RH} 4$ Ventsgroup portant sur « bouche à oreille et recrutement sur les réseaux » avec les réponses de plus de 100000 étudiants et diplômés. En effet, 80\% des interrogés déclarent qu'ils pourraient postuler dans une entreprise uniquement parce qu'ils auraient entendu des échos positifs à son sujet.

D'ici, on remarque la relation inévitable de la marque employeur et le recrutement, cette dernière a une forte influence sur la politique du recrutement.

\section{b. L'e-recrutement / L'inbound recruting}

Le monde des ressources humaines est en train de vivre sa révolution marketing, c'est une opportunité pour se débarrasser des pratiques RH (recrutement) stéréotypés et adopter des pratiques rénovées différenciées par exemple : éviter les annonce de recrutement standards qui utilisent le même jargon.

Les réseaux sociaux constituent un atout pour les entreprises souhaitant avoir une approche directe et attirer les candidats dans un large rayon, rapidement et à moindre coût.

Aujourd'hui, tous ces réseaux servent avant tout des stratégies d'image employeur, comme nous avons pu le voir plus haut.

Ce changement des habitudes et des usages d'internet induit une mutation de la communication corporate et celle du recrutement. La cible (talents) et les entreprises doivent réciproquement s'adapter aux nouvelles pratiques de l'internet communautaire et collaboratif.

L'e-recrutement, dont font partie le recrutement 2.0 et 3.0, permet de réduire les coûts du recrutement, de diffuser rapidement des annonces et d'en simplifier la mise à jour, de partager des informations utiles sur l'entreprise, d'améliorer la qualité du suivi des candidatures et de créer un vivier de candidats.

L'arrivée de ce que l'on appelle "Web 2.0", incluant notamment les réseaux sociaux, a révolutionné le processus d'embauche. Entreprises comme candidats gèrent désormais leur identité numérique à travers leur présence sur des blogs, des réseaux sociaux professionnels. 
Le candidat, comme le recruteur, deviennent ainsi proactifs: le premier en développant son réseau, par exemple, en contactant des "amis d'amis" susceptibles de l'aider ou de le coopter, le second en devenant animateur de communautés autour de la marque employeur.

Le recrutement 3.0 va plus loin en introduisant davantage des techniques issues du Marketing digital, comme l'optimisation du taux de conversion, la notion de Persona candidate4, l'envoi automatisé d'email, tout en mettant en avant la notion d'expérience candidat.

Ces procédés ont été réunis au sein d'une méthodologie à l'efficacité redoutable : l'Inbound Recruiting.

L'Inbound Recruiting est une approche globale qui permet de donner de la cohérence aux différents outils utilisés par les recruteurs, grâce à des publications régulières et de qualité publiées sur le blog carrière et relayées sur les réseaux sociaux afin d'attirer, qualifier, convertir et fidéliser les candidats.

L'inboud recrutement s'inspire du l'inboud marketing, Le concept principal est d'attirer les bonnes personnes à soi et non pas d'aller les chercher (contrairement à l'outbound).

Pour faire simple, le recrutement inbound est donc l'application des techniques d'inbound au recrutement en utilisant les moteurs de recherche, les contenus de blogs et des médias sociaux pour récupérer un maximum de métadonnées sur l'audience et des données sur les internautes potentiellement futurs candidats.

Pour ce faire, vous devriez être le premier résultat que les internautes vont trouver! Pourquoi ? Tout simplement parce que le temps des internautes étant précieux, ils ne vont pas s'amuser à faire 63 pages pour finir par vous trouver. Ils iront jusqu'à la deuxième, peut-être la troisième page mais n'iront jamais plus loin.

Vous devrez faire les yeux doux à Google et jouer sur vos mots-clés (SEO)5 pour qu'il puisse vous envoyer tout en haut de la page.

En effet avec le recrutement inbound on peut mesurer le nombre exact de personnes qui ont été au contact de son message et ceux qui ont cliqué, pour connaitre ce qui a fait le succès (ou l'échec) de la campagne, afin d'en apporter les modifications nécessaires à sa prochaine stratégie.

Parmi les outils utilisés dans l'inbound recruting ou le e-recrutement en général on trouve (selon une étude menée par Régions Jobs sur unéchantillon de 157 recruteurs):

\footnotetext{
${ }^{4}$ Une candidate persona correspond à la représentation fictive que l'on se fait d'un candidat idéal.

5 SEO est l'acronyme de Search Engine Optimization et peut être défini comme l'art de positionner un site, une page web ou une application dans les premiers résultats naturels des moteurs de recherche.
} 
- 53\% de part marché pour Viadeo ;

- $28 \%$ pour LinkedIn ;

- $12 \%$ pour Facebook;

- 6\% des utilisateurs seulement utilise Twitter. Ce réseau est plutôt utilisé pour des recherches de profils plus spécifiques et experts.

In fine Les médias sociaux sont pour les ressources humaines une opportunité de faire un travail de prospect et de terrain ; en effet, le marketing RH au travers des médias sociaux est un marketing d'écoute. Désigné par le terme de « marketing participatif » (ou Social Media Marketing), il consiste à utiliser, identifier et analyser les conversations ainsi qu'à participer et initier des interactions au sein d'une communauté.

\section{Les Strategies De L'e-Recrutement Adoptees Par Quelques Entreprises Multinationales}

Plusieurs entreprises, conscientes de l'importance du marketing $\mathrm{RH}$, ont déjà commencé à prendre la mesure du changement en cours dans le monde notamment à travers le contexte international de crise, la mobilité internationale des talents, l'évolution du management interculturel des hommes, le progrès technologique, etc.

Nous citerons dans ce qui suit le cas de certaines organisations ayant réussi dans le domaine ainsi que certaines de leurs actions.

\section{- Le cas de Sephora}

Sephora, entreprise spécialisée dans le cosmétique, a lancé une marque employeur « l'effet sephora » avec la mise en ligne d'un quizz emploi offrant la possibilité aux internautes de confronter leur profil à la culture de l'entreprise avant de postuler. Le résultat est à la hauteur : en moins de six mois, plus de 10000 internautes avaient effectué le test. Ce qui a permis à Sephora de devenir la première base de CV (45 000 CV).

Elle a également lancé un blog basé sur le principe de transparence permettant à ses collaborateurs « sephorettes » de poster leurs expériences quotidiennes et de poster leurs coups de cœur et leurs coups de gueule.

Avec la mise en ligne du blog $\mathrm{RH}$ et la refonte du site de recrutement leffetsefora.com, les candidatures annuelles sont passées de 35000 à 50000 en deux ans.

\section{- Le cas d'orange}

Orange par exemple propose depuis plus d'un an sa nouvelle application mobile employeur : Orange Jobs, pour trouver un emploi ou un stage dans le Groupe, pour découvrir la diversité des métiers et des parcours proposés par Orange. Orange Jobs c'est « un monde d'opportunités à portée 
de main ». L'application mobile complète un portail de recrutement www.orange.jobs, le site officiel de recrutement.

\section{- Le cas de l'Oréal}

L'Oréal révolutionne une nouvelle fois le monde du recrutement étudiant. Il lance aujourd'hui "Reveal by L'Oréal", un jeu pédagogique en ligne proposé aux étudiants de toutes disciplines et d'horizons différents, désireux d'explorer différentes options de carrière avant de se lancer dans la vie active.

Chaque participant endosse le rôle d'un junior tout juste arrivé chez L'Oréal. Plongé dans un univers Web 2.0 où se mêlent virtuel et réel, il est amené à révéler ses talents en découvrant la culture de l'entreprise et le panorama des métiers existants. Une fois le jeu terminé, il reçoit un bilan personnalisé. Les meilleurs sont invités à venir passer deux jours dans la filiale L'Oréal de leur pays où ils se révèleront encore davantage lors d'activités d'équipe et de rencontres avec les collaborateurs et les recruteurs locaux.

\section{- Le cas de Nespresso}

Selon Arnaud Deschamps, Directeur Général de Nespresso France, « la nouvelle plateforme de recrutement de la marque se base sur le principe de la quête du geste parfait qui consiste à communiquer sur la culture de l'entreprise » selon une interview consultée sur youtube.

Cette identité s'est déclinée en film puis en site internet dans lequel on trouve des conseils pour une candidature parfaite, on y trouve aussi une description de la culture de l'entreprise ainsi qu'une rubrique dédiée au storytelling ${ }^{6}$ qui regroupe toutes les expériences des collaborateurs satisfaits et fidèles.

\section{- Le cas de Deloitte}

Deloitte a en effet, décidé en priorité de travailler sur sa marque employeur en ligne, et a ainsi décidé d'impliquer ses employés sur les médias sociaux pour générer du contenu et du buzz.

- Un site extérieur qui cible parfaitement ses publics, en créant trois catégories distinctes : étudiants, jeunes professionnels et senior pour répondre aux questions et fournir du contenu en segmentant avec précision.

Leur stratégie principale est le témoignage d'employés de Deloitte. Toute l'équipe de recrutement a son profil sur les médias sociaux pour communiquer plus directement et surtout créer des communautés.

\footnotetext{
${ }^{6}$ Technique du marketing qui consiste à raconter son histoire/vécu dans l'entreprise pour convaincre et persuader le futur candidat.
} 
- Des offres géo-localisées Sur la partie offre d'emploi du site, ils ont aussi introduit un concept original ; vous pouvez voir sur une carte où se trouve le job qui vous intéresse, le tout sur une carte Google.

- Twitter est utilisé pour diffuser les offres d'emploi et les événements qu'ils organisent ainsi que pour diffuser les articles de leur blog.

- Facebook se focalise essentiellement sur les candidats potentiels. Le ecommunity manager réagit et interagit aux commentaires des candidats et/ou étudiants en partageant de l'information, en construisant des relations et en tenant informés les fans.

- LinkedIn s'utilise pour le sourcing des candidats les plus seniors ou les plus qualitatifs. Grâce à la nouvelle application « Apply with LinkedIn » les candidats pourront directement postuler aux offres proposées par les entreprises en appuyant simplement sur un bouton.

- Une chaîne Youtube, avec de nombreuses vidéos de témoignages d'employés.

\section{Conclusion}

La marque employeur est l'ensemble d'avantages économiques, fonctionnels et psychologiques, ce concept a gagné l'intérêt des praticiens, avec pour objectif d'améliorer l'attraction des « talents » et la rétention des collaborateurs impliqués.

La marque employeur offre la possibilité de communiquer auprès de ces deux cibles (salariés ou candidats) sur les bénéfices à œuvrer pour cette entreprise et sur ce qui lui permet d'être différente de ses concurrents.

Cette communication doit être réelle et cohérente envers tous ses salariés pour influencer favorablement leurs attitudes et comportements à l'égard de l'organisation.

La marque employeur entrainerait de nombreuses conséquences positives pour l'organisation. En effet, elle permet de créer de la valeur financière pour l'entreprise à travers une diminution de ses coûts associés au turnover et au processus de recrutement.

Aussi, elle améliorerait notamment la qualité des recrutements et le taux de rétention, l'implication, la satisfaction et la motivation des membres du personnel.

De même, elle aide à valoriser les comportements de citoyenneté organisationnelle et participe à plus long terme, à une meilleure performance économique des entreprises qui en ont fait l'une de leurs préoccupations centrales. 
L'objectif de cet article était de montrer d'une part le rôle du marketing RH dans le développement de la marque employeur à l'ère du digital, et d'autre part l'impact de la marque employeur sur l'attractivité de l'entreprise en matière de recrutement.

Nous avons défini la notion de la marque employeur numérique et du e-recrutement et, démontré ainsi la relation qui entretiennent.

In fine l'article a apporté un éclairage sur les nouveaux outils digitaux de recrutement issus du web 2.0. Et finalement nous avons présenté des exemples de stratégie de recrutement 2.0 de quelques entreprises multinationales.

\section{References:}

1. Arnaud, S., Frimousse, S., \& Peretti, J M. (2009). "Gestion personnalisée des ressources humaines : implications et enjeux", Revue : Management Prospective, Edition Management \& Avenir, numéro 28, pp.294-314.

2. Asli, A. \& Baakil, D. (2018). "Le marketing RH : une nouvelle approche de gestion des ressources humaines ", revue Academia, P. 23.

3. Barrow, S. \& Ambler, T. (1996). "The employer brand", Journal of Brand Management. Volume 4 Number 3.

4. Brillet, F. \& Gavoille, F. (2017). Marketing RH. Edition Dunod.

5. Duroni, A. (2011). De la marque employeur aux nouveaux enjeux RH, Ecole des Hautes Etudes Commerciales, thèse soutenue et publiè 2 Mai 2011.

6. Levionnois, M. (1987). Marketing interne et management des hommes. Editions d'Organisation.

7. Liger, P. (2008). Marketing RH : Attirer, intégrer et fidéliser les salariés. Edition Dunod,

8. Liger, P. (2016). le marketing RH comment devenir un employeur attractif, 4ème édition Dunod.

9. Panczuk, S. \& Point, S. (2008). Enjeux et outils du marketing RH : Promouvoir et vendre les ressources humaines. Éditions d'Organisation Groupe Eyrolles, Collection Ressources humaines, pp. 254.

10. Schäfer, N. \& Simon, B. (2011). Mémoire soutenu, quelle importance doivent attribuer les ressources humaines aux réseaux sociaux dans leur stratégie de recrutement, Grande école spécialisé PPA.

11. Wacheux, P. (2005). “l'entreprise est amenée à jouer un rôle de plus en plus social," journal du net.

12. Cabinet d'expertise comptable en ligne, Booster l'attractivité de l'entreprise dans tous les domaines, 2018. https://www.l-expert- 
comptable.com/a/532412-booster-l-attractivite-de-l-entreprise-danstous-les-domaines.html.

13. Clémentine-Lou Henry (2016). L'inbound marketing et le recrutement digital, publié le 11 avril 2016, https://www.digitaweb.com/blog/inbound-marketing-recrutementdigital.

14. Fougerat (2010). La marque employeur est-elle une marque comme les autres ?, consulté dans : http://dircomleblog.canalblog.com/archives/2010/12/06/19664168.ht $\mathrm{ml}$.

15. Guillome vigneron, E-recrutement, recrutement 2.0, recrutement 3.0 : quelles différences ? https://inbound.lasuperagence.com/blog/erecrutement-recrutement-2.0-recrutement-3.0-inbound-recruiting.

16. https://www.coursehero.com/file/p3fjjkc/Selon-une-\%C3\%A9tudemen\%C3\% A9e-par-Regions-Job-sur-un-\%C3\% A9chantillon-de-157recruteurs-les/

17. Jean-Christophe Anna (2011). La Marque Employeur 2.0 est conversationnelle et multicanale, publié le 09 février 2011. https://www.exclusiverh.com/articles/marque-employeur/twuzz-5-lamarque-employeur-2-0-est-conversationnelle-et-multicanal.htm.

18. La Pinta, F. (2011). La marque employeur, http://www.francklapinta.com/definition-marque-employeur/.

19. Laurent brouat, c'est quoi le recrutement inboud? https://lecoledurecrutement.fr/cest-quoi-recrutement-inbound/.

20. Marque employeur et recrutement 2.0 exemples et bonnes pratiques, Région job, file:///C:/Users/hp/Downloads/ebookmarqueemployeur120109085214-phpapp01.pdf.

21. Resultat de l'observatoire hopscotch-viavoice, (2011). Salarié et média sociaux, https://fr.calameo.com/read/00065142529d5c6a23d50.

22. Site de l'Oréal news https://www.loreal.fr/media/pressreleases/2006/mar/1\%E2\%80\%99or\%C3\%A9al-lance-un-conceptin\%C3\%A9dit---lt-i-gt-reveal-by-1\%E2\%80\%99or\%C3\%A9al-lt-igt---le-premier-business-game-multi-m\% $3 \%$ A9tiersr\%C3\%A9v\%C3\%A9lateur-de-talents. 